\title{
Nonradial oscillations of neutron stars with a solid crust
}

\section{Analysis in the relativistic Cowling approximation}

\author{
S. Yoshida ${ }^{1}$ and U. Lee ${ }^{2}$ \\ 1 Centro Multidisciplinar de Astrofísica - CENTRA, Departamento de Física, Instituto Superior Técnico, Av. Rovisco Pais 1 , \\ 1049-001 Lisboa, Portugal \\ e-mail: yoshida@fisica.ist.utl.pt; yoshida@astr.tohoku.ac.jp \\ 2 Astronomical Institute, Graduate School of Science, Tohoku University, Sendai 980-8578, Japan \\ e-mail: lee@astr.tohoku.ac.jp
}

Received 25 June 2002 / Accepted 30 August 2002

\begin{abstract}
Nonradial oscillations of relativistic neutron stars with a solid crust are computed in the relativistic Cowling approximation, in which all metric perturbations are ignored. For the modal analysis, we employ three-component relativistic neutron star models with a solid crust, a fluid core, and a fluid ocean. As a measure for the relativistic effects on the oscillation modes, we calculate the relative frequency difference defined as $\delta \sigma / \sigma \equiv\left(\sigma_{\mathrm{GR}}-\sigma_{\mathrm{N}}\right) / \sigma_{\mathrm{GR}}$, where $\sigma_{\mathrm{GR}}$ and $\sigma_{\mathrm{R}}$ are, respectively, the relativistic and the Newtonian oscillation frequencies. The relative difference $\delta \sigma / \sigma$ takes various values for different oscillation modes of the neutron star model, and the value of $\delta \sigma / \sigma$ for a given mode depends on the physical properties of the models. We find that $|\delta \sigma / \sigma|$ is less than $\sim 0.1$ for most of the oscillation modes we calculate, although there are a few exceptions such as the fundamental (nodeless) toroidal torsional modes in the crust, the surface gravity modes confined in the surface ocean, and the core gravity modes trapped in the fluid core. We also find that the modal properties, represented by the eigenfunctions, are not strongly affected by introducing general relativity. It is however shown that the mode characters of the two interfacial modes, associated with the core/crust and crust/ocean interfaces, have been interchanged between the two through an avoided crossing when we move from Newtonian dynamics to general relativistic dynamics.
\end{abstract}

Key words. relativity - stars: neutron - stars: oscillations

\section{Introduction}

After the discovery of the $r$ mode instability in neutron stars driven by gravitational radiation reaction (Andersson 1998; Friedman \& Morsink 1998), nonradial oscillations of relativistic neutron stars has attracted much wider interest than before in astrophysics (see a recent review, e.g., by Andersson $\&$ Kokkotas 2001). The relativistic formulation of nonradial oscillations of fluid neutron stars was first given by Thorne and his collaborators (e.g., Thorne \& Campolattaro 1967; Price $\&$ Thorne 1969; Thorne 1969a,b), and later on extended to the case of neutron stars with a solid crust in their interior (Schumaker \& Thorne 1983, see also Finn 1990). Since one of the main concerns of these studies was gravitational waves generated by the stellar pulsations (e.g., Thorne 1969a), it was essential to include the perturbations in the metric $g_{\alpha \beta}$ in order to obtain a consistent description of the gravitational waves. However, it was the metric perturbations that made it extremely

Send offprint requests to: $\mathrm{S}$. Yoshida, e-mail: yoshida@astr.tohoku.ac.jp difficult to treat both analytically and numerically the oscillation modes in relativistic stars.

McDermott et al. (1983) introduced a relativistic version of the Cowling approximation, in which all the Eulerian metric perturbations $\delta g_{\alpha \beta}$ are neglected in the relativistic oscillation equations derived by Thorne \& Campolattaro (1967). Under the relativistic Cowling approximation, they calculated the $p$-, $f$-, and $g$-modes of relativistic fluid neutron star models to examine how the oscillation modes depend on the model properties. The relativistic Cowling approximation employed by McDermott et al. (1983) was shown to be good enough to calculate the $p$-modes for non-rotating polytropic stars by Lindblom \& Splinter (1990), who compared the fully relativistic oscillation frequencies to those obtained in the relativistic Cowling approximation. Assuming slow rotation, Yoshida \& Kojima (1997) have also carried out similar computations for the $f$ - and $p$-modes of polytropic models and they confirmed the good applicability of the relativistic Cowling approximation. Quite recently, Yoshida \& Lee (2002) have shown in the relativistic Cowling approximation the existence of the 
relativistic $r$ modes $^{1}$ with $l=m$, which are regarded as a counter part of the Newtonian $r$ modes. We believe that the relativistic Cowling approximation is quite useful to investigate the oscillation modes of relativistic stars, although we understand that under the approximation we cannot discuss inherently relativistic oscillation modes like $w$-modes (see, e.g., Andersson et al. 1996).

In their Newtonian calculations, McDermott et al. (1988) have shown that cold neutron stars with a solid crust can support a rich variety of oscillation modes (see also Strohmayer 1991; Lee \& Strohmayer 1996; Yoshida \& Lee 2001). The purposes of this paper are to calculate the various oscillation modes of relativistic neutron stars with a solid crust in the relativistic Cowling approximation, and to discuss the effects of general relativity on the modal property of the oscillation modes. We regard this paper as an extension of the studies by McDermott et al. (1983) and McDermott et al. (1988). In Sect. 2, we derive relativistic oscillation equations for the solid crust in the relativistic Cowling approximation following the formulation developed by Schumaker \& Thorne (1983) and Finn (1990). Numerical results are discussed in Sect. 3 for nonradial modes of three relativistic neutron star models with a solid crust. Sect. 4 is devoted to discussion and conclusion. In this paper, we use units in which $c=G=1$, where $c$ and $G$ denote the velocity of light and the gravitational constant, respectively.

\section{Formulation}

For modal analysis of neutron stars with a solid crust, we solve general relativistic pulsation equations derived under the relativistic Cowling approximation, in which all the metric perturbations in the matter equations are neglected. We assume that the solid crust is in a strain-free state in the equilibrium unperturbed state and the strain in the crust are generated by small amplitude perturbations superposed on the unperturbed state (see, e.g., Aki \& Richards 1980). For the background unperturbed state, it is therefore possible to assume a static and spherical symmetric state, for which the geometry is given by the following line element:

$$
\begin{aligned}
\left(\mathrm{d} s^{2}\right)_{(0)} & =g_{(0) \alpha \beta} \mathrm{d} x^{\alpha} \mathrm{d} x^{\beta} \\
& =-e^{2 v(r)} \mathrm{d} t^{2}+e^{2 \lambda(r)} \mathrm{d} r^{2}+r^{2} \mathrm{~d} \theta^{2}+r^{2} \sin ^{2} \theta \mathrm{d} \varphi^{2},
\end{aligned}
$$

where the subscript (0) is used to refer to the unperturbed state. The four-velocity of the stellar material in the equilibrium is given by

$u_{(0)}^{\alpha}=e^{-v(r)} t^{\alpha}$,

where $t^{\alpha}$ denotes the timelike Killing vector of the unperturbed spacetime.

In the relativistic Cowling approximation, the basic equations for pulsations are obtained from the energy and momentum conservation laws:

$u^{\alpha} \nabla_{\beta} T_{\alpha}^{\beta}=0, \quad($ energy conservation law $)$

${ }^{1}$ We should note that several studies about the general relativistic $r$-mode have been done (e.g., Kojima 1998; Kojima \& Hosonuma 2000; Lockitch et al. 2001; Yoshida 2001; Ruoff \& Kokkotas 2001). $q_{\gamma}^{\alpha} \nabla_{\beta} T_{\alpha}^{\beta}=0, \quad$ (momentum conservation law)

where $\nabla_{\alpha}$ is the covariant derivative associated with the metric, $T_{\beta}^{\alpha}$ is the energy-momentum tensor, and $q_{\beta}^{\alpha}$ is the projection tensor with respect to the fluid four-velocity $u^{\alpha}$, which is defined by

$q_{\beta}^{\alpha}=\delta_{\beta}^{\alpha}+u^{\alpha} u_{\beta}$,

where $\delta_{\alpha}^{\beta}$ denotes the Kronecker delta.

For relativistic analysis of vibration of the solid crust in neutron stars, we follow the formulation given by Schumaker $\&$ Thorne (1983) and Finn (1990). If we define the rate of shear $\sigma_{\alpha \beta}$ and the rate of expansion $\theta$ as

$\sigma_{\alpha \beta}=\frac{1}{2} q_{\alpha}^{\gamma} q_{\beta}^{\delta}\left(\nabla_{\gamma} u_{\delta}+\nabla_{\delta} u_{\gamma}\right)-\frac{1}{3} q_{\alpha \beta} \theta$

$\theta=q_{\alpha}^{\beta} \nabla_{\beta} u^{\alpha}=\nabla_{\alpha} u^{\alpha}$,

the shear strain tensor $\Sigma_{\alpha \beta}$ is determined, in terms of $\sigma_{\alpha \beta}$ and $\theta$, as a solution of the differential equation given by

$L_{u} \Sigma_{\alpha \beta}=\frac{2}{3} \theta \Sigma_{\alpha \beta}+\sigma_{\alpha \beta}$,

where $L_{u}$ is the Lie derivative along the four-velocity field of the matter (Carter \& Quintana 1972). Once Eq. (8) is solved to give the shear strain tensor $\Sigma_{\alpha \beta}$ in terms of $\sigma_{\alpha \beta}$ and $\theta$ (see Schumaker \& Thorne 1983; Finn 1990), the total stress-energy tensor is given by

$T_{\alpha \beta}=\rho u_{\alpha} u_{\beta}+p q_{\alpha \beta}-2 \mu \Sigma_{\alpha \beta}$,

where $\mu$ stands for the isotropic shear modulus, and we have assumed a Hookean relationship between the shear strain and stress tensors. Here, $\rho$ and $p$ mean the mass-energy density and the isotropic pressure, respectively. Note that $\Sigma_{(0) \alpha \beta}=0$ since we have assumed the strain-free state in the equilibrium of the star.

To discuss nonradial oscillations of a star, we employ a Lagrangian perturbation formalism (see, e.g., Friedman \& Schutz 1975; Friedman 1978), in which a Lagrangian displacement vector is introduced to connect fluid elements in the equilibrium state to the corresponding elements in the perturbed state. In this formalism, the Lagrangian change $\Delta Q$ in a quantity is related to its Eulerian change $\delta Q$ by

$\Delta Q=\delta Q+L_{\zeta} Q$

where $L_{\zeta}$ denotes the Lie derivative along the displacement vector $\zeta^{\alpha}$. If we apply this formalism to oscillations of relativistic stars, we have, for example,

$\Delta g_{\alpha \beta}=\delta g_{\alpha \beta}+\nabla_{\alpha} \zeta_{\beta}+\nabla_{\beta} \zeta_{\alpha}$

and

$\Delta u^{\alpha}=\frac{1}{2} u^{\alpha} u^{\beta} u^{\gamma} \Delta g_{\beta \gamma}$

The Eulerian perturbation in the velocity field $\delta \hat{u}^{\beta} \equiv q_{(0)}{ }_{\beta}^{\alpha} \delta u^{\beta}$ is then given by

$\delta \hat{u}^{\alpha}=q_{(0)}^{\alpha}\left(L_{u_{(0)}} \zeta\right)^{\beta}$. 
Notice that $\delta g_{\alpha \beta}=0$ and hence $\delta \hat{u}^{\alpha}=\delta u^{\alpha}$ in the relativistic Cowling approximation. Because the background state is in a hydrostatic equilibrium, the time dependence of all the perturbed quantities can be given by $e^{i \sigma t}$, where $\sigma$ is a constant frequency measured by an inertial observer at the spatial infinity. The relation between the Lagrangian displacement $\zeta^{\alpha}$ and the velocity perturbation $\delta \hat{u}^{\alpha}$ is then given as an algebraic equation:

$\delta \hat{u}^{\alpha}=i \sigma e^{-v(r)} \zeta^{\alpha}$.

Note that the gauge freedom in $\zeta^{\alpha}$ has been used to demand the relation $u_{\alpha} \zeta^{\alpha}=0$. The velocity field of a neutron star in a perturbed state may be written as

$u^{\alpha}=u_{(0)}^{\alpha}+\eta \delta \hat{u}^{\alpha}+O\left(\eta^{2}\right)$,

where $\eta$ is a small expansion parameter introduced for our convenience. The mass-energy density $\rho$ and the pressure $p$ in the perturbed state are also given by

$\rho=\rho_{(0)}+\eta\left(\rho_{(0)}+p_{(0)}\right)\left(\frac{\Gamma \delta p}{p_{(0)}}-\zeta^{\alpha} A_{\alpha}\right)+O\left(\eta^{2}\right)$,

$p=p_{(0)}+\eta \delta p+O\left(\eta^{2}\right)$

where $A_{\alpha}$ is the relativistic Schwarzschild discriminant defined as

$A_{\alpha}=\frac{1}{\rho_{(0)}+p_{(0)}} \nabla_{\alpha} \rho_{(0)}-\frac{1}{\Gamma p_{(0)}} \nabla_{\alpha} p_{(0)}$,

and $\Gamma$ is the adiabatic index defined as

$\Gamma=\frac{\rho_{(0)}+p_{(0)}}{p_{(0)}}\left(\frac{\partial p_{(0)}}{\partial \rho_{(0)}}\right)_{\mathrm{ad}}$.

To derive Eq. (16), we have used the adiabatic condition for the perturbations:

$\Delta p=\frac{\Gamma p_{(0)}}{\rho_{(0)}+p_{(0)}} \Delta \rho$.

Assuming spherical symmetry of the background spacetime, we may expand the perturbations in terms of appropriate tensor spherical harmonic functions. The Lagrangian displacement, $\zeta^{k}$ and the pressure perturbation, $\delta p /\left(\rho_{(0)}+p_{(0)}\right)$ can be written as

$\zeta^{r}=r S_{l}(r) Y_{l}^{m}(\theta, \varphi) e^{i \sigma t}$

$\zeta^{\theta}=\left(H_{l}(r) \frac{\partial Y_{l}^{m}(\theta, \varphi)}{\partial \theta}-T_{l^{\prime}}(r) \frac{1}{\sin \theta} \frac{\partial Y_{l^{\prime}}^{m}(\theta, \varphi)}{\partial \varphi}\right) e^{i \sigma t}$,

$\zeta^{\varphi}=\frac{1}{\sin ^{2} \theta}\left(H_{l}(r) \frac{\partial Y_{l}^{m}(\theta, \varphi)}{\partial \varphi}+T_{l^{\prime}}(r) \sin \theta \frac{\partial Y_{l^{\prime}}^{m}(\theta, \varphi)}{\partial \theta}\right) e^{i \sigma t}$,

$\frac{\delta p}{\rho_{(0)}+p_{(0)}}=\delta U_{l}(r) Y_{l}^{m}(\theta, \varphi) e^{i \sigma t}$,

where $Y_{l}^{m}$ is a spherical harmonic function (Regge \& Wheeler 1957; Thorne 1980).

By substituting Eqs. (9), (15)-(17), together with the perturbations defined by Eqs. (21) to (24), into Eqs. (3) and (4), and collecting the terms proportional to the small expansion parameter $\eta$, we obtain a system of perturbation equations for the solid crust:

$r \frac{\mathrm{d} z_{1}}{\mathrm{~d} r}=-\left(1+\frac{2 \alpha_{2}}{\alpha_{3}}+U_{2}\right) z_{1}+\frac{1}{\alpha_{3}} z_{2}+\frac{\alpha_{2}}{\alpha_{3}} l(l+1) z_{3}$,

$r \frac{\mathrm{d} z_{2}}{\mathrm{~d} r}=\left\{\left(-3-U_{2}+U_{1}-e^{2 \lambda} c_{1} \bar{\sigma}^{2}\right) V_{1}\right.$

$\left.+\frac{4 \alpha_{1}}{\alpha_{3}}\left(3 \alpha_{2}+2 \alpha_{1}\right)\right\} z_{1}+\left(V_{2}-4 \frac{\alpha_{1}}{\alpha_{3}}\right) z_{2}$

$+\left\{V_{1}-2 \alpha_{1}\left(1+\frac{2 \alpha_{2}}{\alpha_{3}}\right)\right\} l(l+1) z_{3}+e^{2 \lambda} l(l+1) z_{4}$,

$r \frac{\mathrm{d} z_{3}}{\mathrm{~d} r}=-e^{2 \lambda} z_{1}+\frac{e^{2 \lambda}}{\alpha_{1}} z_{4}$,

$r \frac{\mathrm{d} z_{4}}{\mathrm{~d} r}=-\left(-V_{1}+6 \Gamma \frac{\alpha_{1}}{\alpha_{3}}\right) z_{1}-\frac{\alpha_{2}}{\alpha_{3}} z_{2}$

$$
-\left\{c_{1} \bar{\sigma}^{2} V_{1}+2 \alpha_{1}-\frac{2 \alpha_{1}}{\alpha_{3}}\left(\alpha_{2}+\alpha_{3}\right) l(l+1)\right\} z_{3}
$$$$
-\left(3+U_{2}-V_{2}\right) z_{4},
$$

$r \frac{\mathrm{d} z_{5}}{\mathrm{~d} r}=\frac{e^{2 \lambda}}{\alpha_{1}} z_{6}$,

$r \frac{\mathrm{d} z_{6}}{\mathrm{~d} r}=-\left(3+U_{2}-V_{2}\right) z_{6}$

$$
-\left\{c_{1} \bar{\sigma}^{2} V_{1}-\alpha_{1}\left(l^{\prime}+1\right)\left(l^{\prime}-2\right)\right\} z_{5},
$$

where the dependent variables $z_{1}$ to $z_{6}$ are defined as

$z_{1}=S_{l}(r)$

$$
\begin{aligned}
z_{2}= & 2 \alpha_{1} e^{-\lambda} \frac{\mathrm{d}}{\mathrm{d} r}\left(r e^{\lambda} S_{l}(r)\right)+\left(\Gamma-\frac{2}{3} \alpha_{1}\right) \\
& \times\left\{\frac{e^{-\lambda}}{r^{2}} \frac{\mathrm{d}}{\mathrm{d} r}\left(r^{3} e^{\lambda} S_{l}(r)\right)-l(l+1) H_{l}(r)\right\},
\end{aligned}
$$

$z_{3}=H_{l}(r)$,

$z_{4}=\alpha_{1}\left(e^{-2 \lambda} r \frac{\mathrm{d} H_{l}(r)}{\mathrm{d} r}+S_{l}(r)\right)$,

$z_{5}=T_{l^{\prime}}(r)$

$z_{6}=\alpha_{1} e^{-2 \lambda} r \frac{\mathrm{d} T_{l^{\prime}}(r)}{\mathrm{d} r}$

and the various quantities which appear in the coefficients are

$\alpha_{1}=\frac{\mu}{p_{(0)}}, \quad \alpha_{2}=\Gamma-\frac{2}{3} \alpha_{1}, \quad \alpha_{3}=\Gamma+\frac{4}{3} \alpha_{1}$,

$V_{1}=\left(1+\frac{\rho_{(0)}}{\varepsilon p_{(0)}}\right) r \frac{\mathrm{d} v}{\mathrm{~d} r}, \quad V_{2}=\frac{\rho_{(0)}}{\varepsilon p_{(0)}} r \frac{\mathrm{d} v}{\mathrm{~d} r}$,

$$
U_{1}=\left(\frac{\mathrm{d} v}{\mathrm{~d} r}\right)^{-1} \frac{\mathrm{d}}{\mathrm{d} r}\left(r \frac{\mathrm{d} v}{\mathrm{~d} r}\right), \quad U_{2}=r \frac{\mathrm{d} \lambda}{\mathrm{d} r},
$$

$c_{1}=\varepsilon \frac{M}{R^{3}} r e^{-2 \varepsilon v}\left(\frac{\mathrm{d} v}{\mathrm{~d} r}\right)^{-1}$,

$e^{2 \lambda}=\left(1-\varepsilon \frac{2 M(r)}{r}\right)^{-1}, \quad M(r)=\int_{0}^{r} 4 \pi r^{2} \rho_{(0)} \mathrm{d} r$,

$r \frac{\mathrm{d} v}{\mathrm{~d} r}=\varepsilon e^{2 \lambda}\left(4 \pi r^{2} \varepsilon p_{(0)}+\frac{M(r)}{r}\right)$,

$r \frac{\mathrm{d} \lambda}{\mathrm{d} r}=\varepsilon e^{2 \lambda}\left(4 \pi r^{2} \rho_{(0)}-\frac{M(r)}{r}\right)$. 
Here, $M=M(R)$ and $R$ are the mass and the radius of the star, and $\bar{\sigma}=\sigma \sqrt{R^{3} / M}$ is the dimensionless frequency. In Eqs. (38)-(41), we have introduced a factor $\varepsilon$ to indicate the hidden factor $1 / c^{2}$ that represents the strength of the general relativistic degree. Fully relativistic oscillation equations for the solid crust are regained for $\varepsilon=1$, and the Newtonian oscillation equations are obtained in the limit of $\varepsilon \rightarrow 0$, which are the same as those derived by McDermott et al. (1988). We can see that Eqs. (25)-(28) are decoupled from Eqs. (29), (30) because the background spacetime is spherical symmetric. The former describe spheroidal (or polar parity) oscillations, and the latter toroidal (or axial parity) ones.

In the fluid regions, the oscillation equations to be solved are given by

$r \frac{\mathrm{d} y_{1}}{\mathrm{~d} r}=-\left(3-\frac{V_{1}}{\Gamma}+U_{2}\right) y_{1}-\left(\frac{V_{1}}{\Gamma}-\frac{l(l+1)}{c_{1} \bar{\sigma}^{2}}\right) y_{2}$,

$r \frac{\mathrm{d} y_{2}}{\mathrm{~d} r}=\left(e^{2 \lambda} c_{1} \bar{\sigma}^{2}+r A_{r}\right) y_{1}-\left(U+r A_{r}\right) y_{2}$,

where

$y_{1}=S_{l}(r), \quad y_{2}=\left(r \frac{\mathrm{d} v}{\mathrm{~d} r}\right)^{-1} \delta U_{l}(r)=c_{1} \bar{\sigma}^{2} H_{l}$.

Note that Eqs. (42) and (43), which come from Eqs. (25)-(28) in the limit of $\mu \rightarrow 0$, are essentially the same as those derived by McDermott et al. (1983) for spheroidal oscillation modes. Equations (29)-(30), which describe toroidal torsional oscillations, become trivial in the limit of $\mu \rightarrow 0$ because there is no restoring force that is responsible for the toroidal torsional oscillations. To calculate the spheroidal modes, Eqs. (25)-(28) are integrated in the solid crust, and Eqs. (42), (43) in the fluid core and the surface ocean. For the toroidal torsional modes, Eqs. (29), (30) should be solved only in the solid crust.

For the spheroidal modes the outer boundary condition is given at the stellar surface $(r=R)$ by $\Delta p=0$, which reduces to

$y_{1}-y_{2}=0$,

and the inner boundary condition is the regularity condition at the stellar center given by

$c_{1} \bar{\sigma}^{2} y_{1}-l y_{2}=0$.

The jump conditions at the interfaces between the fluid and the solid regions are given by the continuity conditions, across the perturbed interface, of the stress $P_{\alpha}$ on the interface, where

$P_{\alpha}=\left(p q_{\alpha \beta}-2 \mu \Sigma_{\alpha \beta}\right) N^{\beta}$,

and $N^{\beta}$ is the unit vector normal to the perturbed interface (see, e.g., Finn 1990). The normal form of the core/crust or crust/ocean interface is given by $\mathrm{d}\left(r-\eta \zeta^{r}\right)=n_{\alpha} \mathrm{d} x^{\alpha}$ for the radial coordinate of the perturbed interface given by $r=R_{i}+\eta \zeta^{r}$, where $R_{i}$ is the radius of the core/crust or crust/ocean interface in the unperturbed state. Thus, the corresponding unit normal one-form $N_{\alpha}$ have the components as follows:

$N_{t}=-e^{\lambda} \eta i \sigma r S_{l}(r) Y_{l}^{m}(\theta, \varphi) e^{i \sigma t}+O\left(\eta^{2}\right)$,

$N_{r}=e^{\lambda}+O\left(\eta^{2}\right)$,
Table 1. Neutron star models.

\begin{tabular}{cccccc}
\hline \hline Model & $M\left(M_{\odot}\right)$ & $R(\mathrm{~km})$ & $\rho_{\mathrm{c}}\left(\mathrm{g} \mathrm{cm}^{3}\right)$ & $T_{\mathrm{c}}(\mathrm{K})$ & $G M /\left(c^{2} R\right)$ \\
\hline NS05T7 & 0.503 & 9.839 & $9.44 \times 10^{14}$ & $1.03 \times 10^{7}$ & $7.54 \times 10^{-2}$ \\
NS05T8 & 0.503 & 9.785 & $9.44 \times 10^{14}$ & $9.76 \times 10^{7}$ & $7.59 \times 10^{-2}$ \\
NS13T8 & 1.326 & 7.853 & $3.63 \times 10^{15}$ & $1.05 \times 10^{8}$ & $2.49 \times 10^{-1}$ \\
\hline
\end{tabular}

$N_{\theta}=-e^{\lambda} \eta r S_{l}(r) \frac{\partial Y_{l}^{m}(\theta, \varphi)}{\partial \theta} e^{i \sigma t}+O\left(\eta^{2}\right)$,

$N_{\varphi}=-e^{\lambda} \eta r S_{l}(r) \frac{\partial Y_{l}^{m}(\theta, \varphi)}{\partial \varphi} e^{i \sigma t}+O\left(\eta^{2}\right)$.

The jump conditions for the spheroidal modes at the interface are then given by

$y_{1}=z_{1}, \quad V_{1}\left(y_{1}-y_{2}\right)=z_{2}, \quad z_{4}=0$.

For the toroidal torsional modes, the boundary conditions are applied at the top and the bottom of the solid crust, and they are

$z_{6}=0$.

By imposing the boundary conditions given above, we can solve our basic equations as an eigenvalue problem with respect to the eigenvalue $\bar{\sigma}$. Here, we employ a Henyey type relaxation method to obtain numerical solutions to our basic equations (see, e.g., Unno et al. 1989).

\section{Numerical results}

Neutron star models that we use in this paper are the same as those used in the modal analysis by McDermott et al. (1988). These models are taken from the evolutionary sequences for cooling neutron stars calculated by Richardson et al. (1982), where the envelope structure is constructed by following Gudmundsson et al. (1983). They are composed of a fluid core, a solid crust and a surface fluid ocean. The interior temperature is finite and is not constant as a function of the radial distance $r$. The models are not barotropic and the Schwarzschild discriminant $|A|$ has finite values in the interior of the star. The models we use are called NS05T7, NS05T8, and NS13T8, and their physical properties such as the total mass $M$, the radius $R$, the central density $\rho_{\mathrm{c}}$, the central temperature $T_{\mathrm{c}}$ and the relativistic factor $G M / c^{2} R$ are summarized in Table 1 (for other quantities, see McDermott et al. 1988).

To classify the various oscillation modes of the three component models, we use almost the same nomenclature as that employed by McDermott et al. (1988). We let $p_{k}$ refer to the acoustic modes of the $k$ th overtone. The internal gravity modes confined in the surface ocean are denoted as $g_{k}^{\mathrm{s}}$ and those in the fluid core as $g_{k}^{\mathrm{c}}$, where $k$ indicates the overtone number. The eigenfrequencies of the $f$ modes are usually found between the $p_{1}$ and $g_{1}$ modes. Associated with the fluid-solid interfaces in the models, there are two interfacial modes, which we denote as $i_{1}$ and $i_{2}$ such that $\sigma\left(i_{1}\right) \leq \sigma\left(i_{2}\right)$. The $s_{k}$ modes are spheroidal shear dominated modes of the $k$ th overtone, the amplitudes of which are strongly confined in the solid crust. The $t_{k}$ modes are toroidal shear dominated modes of the $k$ th overtone propagating only in the solid crust. Note that the $p_{k^{-}}, g_{k^{-}}, f-, i_{1(2)}$, and 
Table 2. Eigenfrequencies $\bar{\sigma}(l=2)$ of NS05T7.

\begin{tabular}{cllr}
\hline \hline mode & $\bar{\sigma}_{\mathrm{N}}$ & $\bar{\sigma}_{\mathrm{GR}}$ & $\delta \sigma / \sigma$ \\
\hline (Spheroidal) & & & \\
$g_{1}^{c}$ & $1.998 \times 10^{-5}$ & $1.625 \times 10^{-5}$ & -0.230 \\
$g_{2}^{s}$ & $2.037 \times 10^{-3}$ & $1.760 \times 10^{-3}$ & -0.158 \\
$g_{1}^{s}$ & $3.230 \times 10^{-3}$ & $2.787 \times 10^{-3}$ & -0.159 \\
$i_{1}$ & $8.282 \times 10^{-3}$ & $7.499 \times 10^{-3}$ & -0.104 \\
$i_{2}$ & $1.029 \times 10^{-1}$ & $9.717 \times 10^{-2}$ & -0.059 \\
$s_{1}$ & $3.093 \times 10^{-1}$ & $3.159 \times 10^{-1}$ & 0.021 \\
$s_{2}$ & $5.556 \times 10^{-1}$ & $5.548 \times 10^{-1}$ & -0.001 \\
$f$ & $1.886 \times 10^{0}$ & $1.823 \times 10^{0}$ & -0.034 \\
$p_{1}$ & $4.038 \times 10^{0}$ & $3.978 \times 10^{0}$ & -0.015 \\
$p_{2}$ & $4.736 \times 10^{0}$ & $4.710 \times 10^{0}$ & -0.006 \\
$($ Toroidal $)$ & & & \\
$t_{0}$ & $4.019 \times 10^{-2}$ & $3.619 \times 10^{-2}$ & -0.111 \\
$t_{1}$ & $3.286 \times 10^{-1}$ & $3.279 \times 10^{-1}$ & -0.002 \\
$t_{2}$ & $5.633 \times 10^{-1}$ & $5.609 \times 10^{-1}$ & -0.004 \\
$t_{3}$ & $7.346 \times 10^{-1}$ & $7.355 \times 10^{-1}$ & 0.001 \\
\hline
\end{tabular}

$s_{k}$ modes are classified as spheroidal modes while the $t_{k}$ modes as toroidal modes.

In the Newtonian limit of $\varepsilon \rightarrow 0$, we calculate various oscillation modes with $l=2$ for the three neutron star models NS05T7, NS05T8, and NS13T8, and tabulate the Newtonian eigenfrequencies, which we denote as $\bar{\sigma}_{\mathrm{N}}$, in Tables 2 through 4 . These tables confirm that the Newtonian frequencies obtained in this paper are in good agreement with those computed by McDermott et al. (1988). Assuming $\varepsilon=$ 1 , we also compute the corresponding relativistic oscillation modes with $l=2$, and tabulate the relativistic eigenfrequencies, which we denote as $\bar{\sigma}_{\mathrm{GR}}$, in Tables 2 through 4 , in which the relative frequency differences defined as $\delta \sigma / \sigma \equiv\left(\sigma_{\mathrm{GR}}-\right.$ $\left.\sigma_{\mathrm{N}}\right) / \sigma_{\mathrm{GR}}$ are also given. The relative difference $\delta \sigma / \sigma$ takes various values for different oscillation modes, and the value of $\delta \sigma / \sigma$ of a given mode is also dependent on the physical properties of the neutron star models. We find $|\delta \sigma / \sigma| \lesssim 0.1$ for most of the oscillation modes we calculate. The elastic $s_{k}$ and $t_{k}$ modes with $k \geq 1$ have particularly small values of $|\delta \sigma / \sigma|$, which are at most a few percent. However, there are some exceptions, and the values of $\delta \sigma / \sigma$ become as large as -0.45 for the $t_{0}$ modes and $\sim-1$ for the $g^{\mathrm{s}}$ - and $g^{\mathrm{c}}$-modes for the model NS13T8, which is the most compact one among the three. If we consider the gravitational redshift as one of the general relativistic effects that are responsible for the difference from the Newtonian oscillation frequency, we may have $\tilde{\sigma}_{\mathrm{GR}}=e^{\nu(r)} \sigma_{\mathrm{N}}$ and hence $\left(\tilde{\sigma}_{\mathrm{GR}}-\sigma_{\mathrm{N}}\right) / \tilde{\sigma}_{\mathrm{GR}}=1-e^{-v(r)}$, which is negative since $v(r)<0$. Although this estimation gives a consistent result for the surface $g^{\mathrm{s}}$ modes confined in the surface ocean and for the core $g^{\mathrm{c}}$ modes trapped in the fluid core, it does not always give good estimations of the difference as suggested by the tables. In fact, we have positive $\delta \sigma / \sigma$ for some oscillation modes. This is understandable, however, since the eigenfunctions usually possess large radial extent in the interior of stars, and the eigenfrequencies are dependent on the equilibrium quantities which contain terms and factors which are absent in the Newtonian oscillation equations.
Table 3. Eigenfrequencies $\bar{\sigma}(l=2)$ of NS05T8.

\begin{tabular}{cllr}
\hline \hline mode & $\bar{\sigma}_{\mathrm{N}}$ & \multicolumn{1}{l}{$\bar{\sigma}_{\mathrm{GR}}$} & $\delta \sigma / \sigma$ \\
\hline (Spheroidal) & & & \\
$g_{1}^{c}$ & $2.248 \times 10^{-4}$ & $1.821 \times 10^{-4}$ & -0.234 \\
$g_{2}^{s}$ & $1.297 \times 10^{-2}$ & $1.102 \times 10^{-2}$ & -0.177 \\
$g_{1}^{s}$ & $1.507 \times 10^{-2}$ & $1.289 \times 10^{-2}$ & -0.169 \\
$i_{1}$ & $5.346 \times 10^{-2}$ & $5.894 \times 10^{-2}$ & 0.093 \\
$i_{2}$ & $1.002 \times 10^{-1}$ & $9.236 \times 10^{-2}$ & -0.085 \\
$s_{1}$ & $3.251 \times 10^{-1}$ & $3.299 \times 10^{-1}$ & 0.014 \\
$s_{2}$ & $5.755 \times 10^{-1}$ & $5.746 \times 10^{-1}$ & -0.002 \\
$f$ & $1.873 \times 10^{0}$ & $1.811 \times 10^{0}$ & -0.035 \\
$p_{1}$ & $4.102 \times 10^{0}$ & $4.027 \times 10^{0}$ & -0.019 \\
$p_{2}$ & $4.766 \times 10^{0}$ & $4.747 \times 10^{0}$ & -0.004 \\
$($ Toroidal $)$ & & & \\
$t_{0}$ & $3.999 \times 10^{-2}$ & $3.600 \times 10^{-2}$ & -0.111 \\
$t_{1}$ & $3.419 \times 10^{-1}$ & $3.410 \times 10^{-1}$ & -0.003 \\
$t_{2}$ & $5.841 \times 10^{-1}$ & $5.812 \times 10^{-1}$ & -0.005 \\
$t_{3}$ & $8.148 \times 10^{-1}$ & $8.128 \times 10^{-1}$ & -0.002 \\
\hline
\end{tabular}

Table 4. Eigenfrequencies $\bar{\sigma}(l=2)$ of NS13T8.

\begin{tabular}{cllr}
\hline \hline mode & $\bar{\sigma}_{\mathrm{N}}$ & \multicolumn{1}{l}{$\bar{\sigma}_{\mathrm{GR}}$} & $\delta \sigma / \sigma$ \\
\hline (Spheroidal) & & & \\
$g_{1}^{c}$ & $1.269 \times 10^{-4}$ & $6.017 \times 10^{-5}$ & -1.109 \\
$g_{2}^{s}$ & $6.115 \times 10^{-3}$ & $3.126 \times 10^{-3}$ & -0.956 \\
$g_{1}^{s}$ & $7.548 \times 10^{-3}$ & $3.905 \times 10^{-3}$ & -0.933 \\
$i_{1}$ & $1.862 \times 10^{-2}$ & $2.357 \times 10^{-2}$ & 0.210 \\
$i_{2}$ & $3.326 \times 10^{-2}$ & $2.985 \times 10^{-2}$ & -0.114 \\
$s_{1}$ & $4.512 \times 10^{-1}$ & $4.528 \times 10^{-1}$ & 0.004 \\
$s_{2}$ & $7.704 \times 10^{-1}$ & $7.685 \times 10^{-1}$ & -0.002 \\
$f$ & $1.434 \times 10^{0}$ & $1.249 \times 10^{0}$ & -0.148 \\
$p_{1}$ & $3.967 \times 10^{0}$ & $3.038 \times 10^{0}$ & -0.306 \\
$p_{2}$ & $5.511 \times 10^{0}$ & $4.602 \times 10^{0}$ & -0.197 \\
$($ Toroidal $)$ & & & \\
$t_{0}$ & $1.897 \times 10^{-2}$ & $1.309 \times 10^{-2}$ & -0.449 \\
$t_{1}$ & $4.534 \times 10^{-1}$ & $4.533 \times 10^{-1}$ & -0.000 \\
$t_{2}$ & $7.727 \times 10^{-1}$ & $7.695 \times 10^{-1}$ & -0.004 \\
$t_{3}$ & $1.105 \times 10^{0}$ & $1.100 \times 10^{0}$ & -0.004 \\
\hline
\end{tabular}

For the various oscillation modes of NS13T8, we display the displacement vector $\zeta^{\alpha}$ versus the radial coordinates $r / R$ or $\log (1-r / R)$ in Figs. 1 through 8 , where the amplitude normalization of the eigenfunctions is given by $y_{1}=1$ at $r=R$ for the spheroidal modes and $z_{5}=1$ at $r=R_{\text {out }}$ for the toroidal modes with $R_{\text {out }}$ being the radius of the ocean-crust interface. Notice that for the $g^{\mathrm{c}}$ modes, the normalization condition $H_{l}\left(R_{\text {in }}\right)=1$ is adapted because the modes are very insensitive to the behavior of the eigenfunctions in the surface ocean, where $R_{\text {in }}$ is the radius at the bottom of the crust. The eigenfunctions obtained in the Newtonian calculations are also plotted for comparison in each of the figures. From Figs. 1 through 8, we can see that the basic properties of the eigenfunctions of the relativistic modes are not very much different from those of the corresponding Newtonian modes, except for the interfacial modes (see the next paragraph). In this sense, we can say that general relativity does not bring about any essential changes in the modal properties represented by the eigenfunctions. 


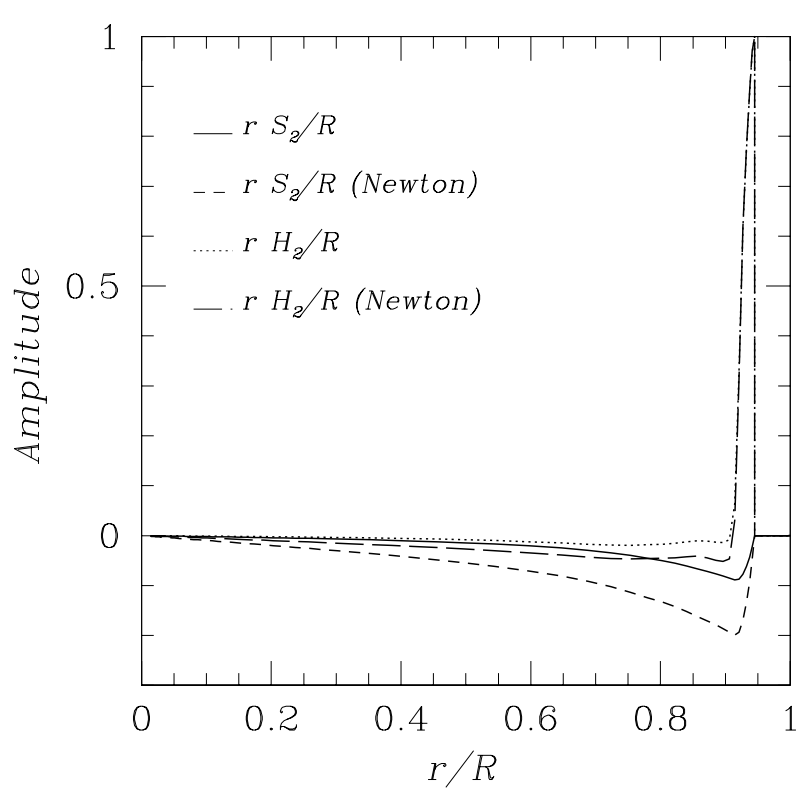

Fig. 1. Displacement vectors $r S_{2} / R$ and $r H_{2} / R$ of the $g_{1}^{\text {c }}$ mode for the model NS13T8, given as a function of $r / R$. Here, the normalization of the eigenfunction is chosen as $H_{2}\left(R_{\text {in }}\right)=1$, where $R_{\text {in }}$ is the radius at the bottom of the crust. The Newtonian eigenfunctions are shown as well as the relativistic ones.

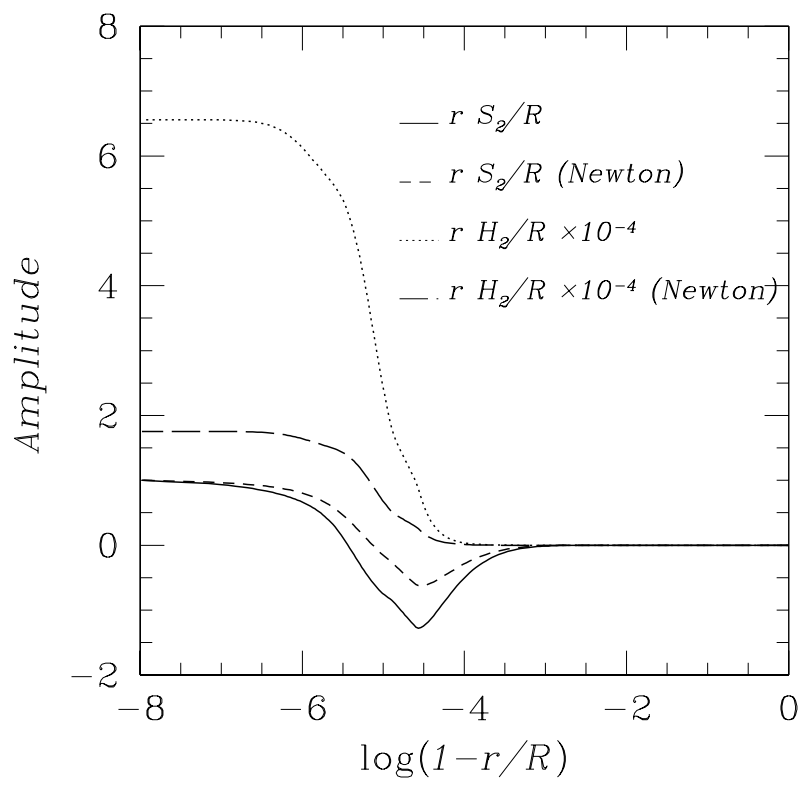

Fig. 2. Displacement vectors $r S_{2} / R$ and $r H_{2} / R$ of the $g_{1}^{s}$ mode for the model NS13T8, given as a function of $\log (1-r / R)$. Here, the normalization of the eigenfunction is chosen as $S_{2}(R)=1$. The Newtonian eigenfunctions are shown as well as the relativistic ones.

As shown by Figs. 3 and 4, the relativistic eigenfunctions of the interfacial modes $i_{1}$ and $i_{2}$ are at first sight quite different from the Newtonian eigenfunctions. Since the eigenfunctions of the relativistic $i_{1(2)}$ mode are rather similar to those of the Newtonian $i_{2(1)}$ mode, it is tempting to make a guess that the modal properties have been exchanged between the two modes when we move from Newtonian dynamics to relativistic dynamics. In their Newtonian calculation, McDermott et al. (1988) showed that the properties of the $i$ modes are very

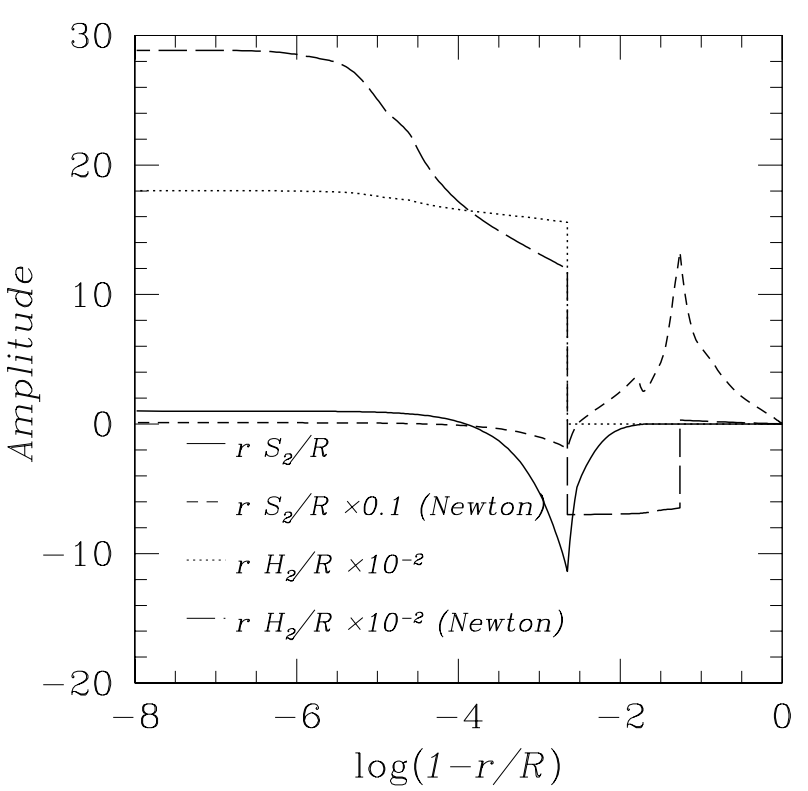

Fig. 3. Same as Fig. 2 but for the $i_{1}$ mode.

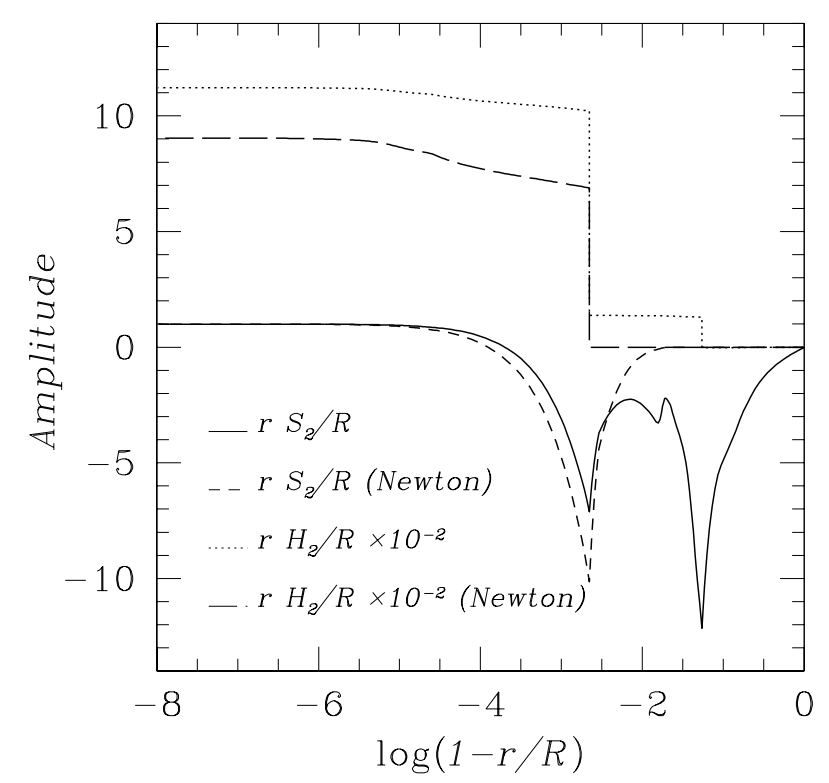

Fig. 4. Same as Fig. 3 but for the $i_{2}$ mode.

sensitive to the changes in the physical quantities near the interfaces, and mentioned a numerical experiment in which they computed the two interfacial modes by artificially reducing the bulk modulus $\mu$ and found an avoided crossing between the two modes through which the modal characters are interchanged with each other. Inspired by this report, we have carried out a numerical experiment, calculating the two interfacial modes as a function of $\varepsilon$ for the model NS13T8. Plotting the frequencies of the two modes versus $\varepsilon$ in Fig. 9, we confirm the occurrence of an avoided crossing between the two modes at $\varepsilon \sim 0.67$, through which the modal characters of the two have been exchanged with each other. As $\varepsilon$ increases from $\varepsilon=0$ (Newtonian limit), the effective local shear modulus $\mu$ in the solid crust decreases because of the gravitational redshift effects, as a result of which the two interfacial modes experience the avoided 


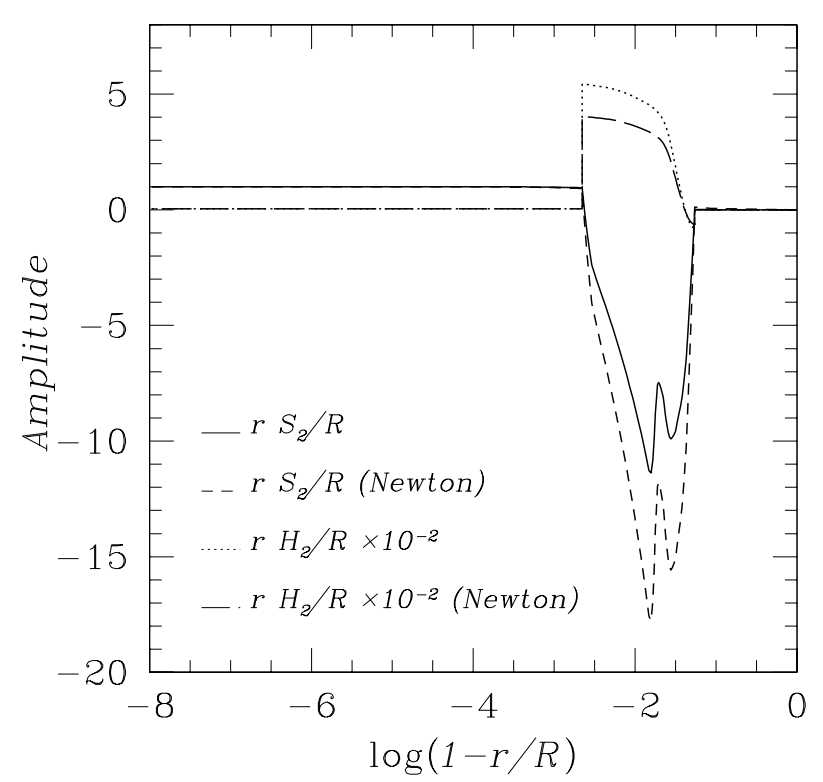

Fig. 5. Same as Fig. 4 but for the $s_{1}$ mode.

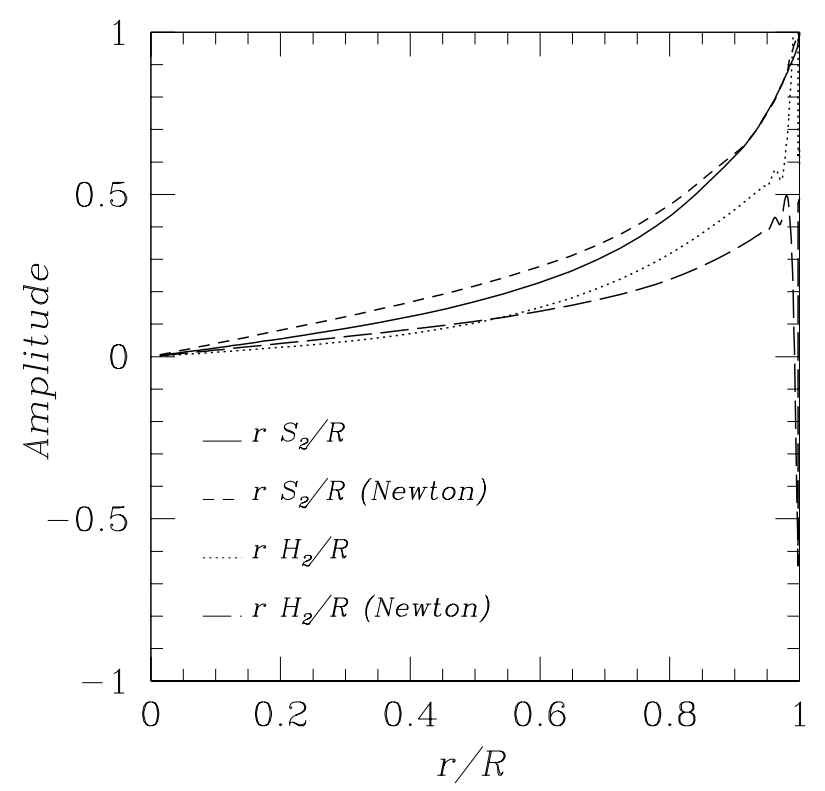

Fig. 6. Displacement vectors $r S_{2} / R$ and $r H_{2} / R$ of the $f$ mode for the model NS13T8, given as a function of $r / R$. Here, the normalization of the eigenfunction is chosen as $S_{2}(R)=1$. The Newtonian eigenfunctions are shown as well as the relativistic ones.

crossing when we move from Newtonian dynamics to relativistic dynamics.

\section{Conclusion}

In this paper, we have calculated a variety of oscillation modes of relativistic neutron stars with a solid crust in the relativistic Cowling approximation, in which all metric perturbations are ignored. We find $|\delta \sigma / \sigma|$ is less than $\sim 0.1$ for most of the oscillation modes with $l=2$, although there are some exceptions such as the surface gravity modes, the core gravity modes, and the nodeless toroidal torsional modes. We also find that the essential modal properties represented by the eigenfunctions are

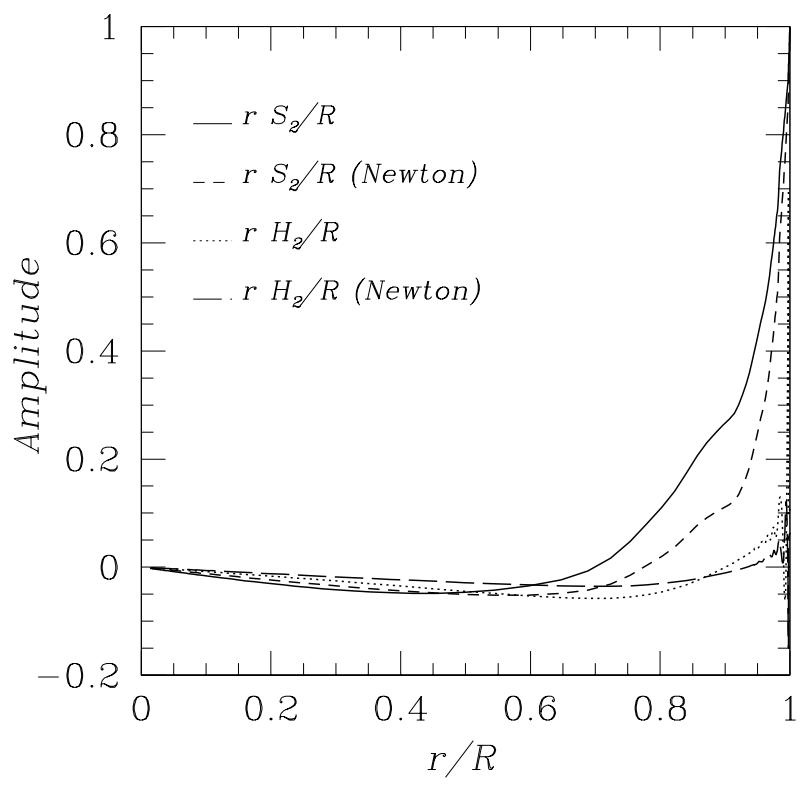

Fig. 7. Same as Fig. 6 but for the $p_{1}$ mode.

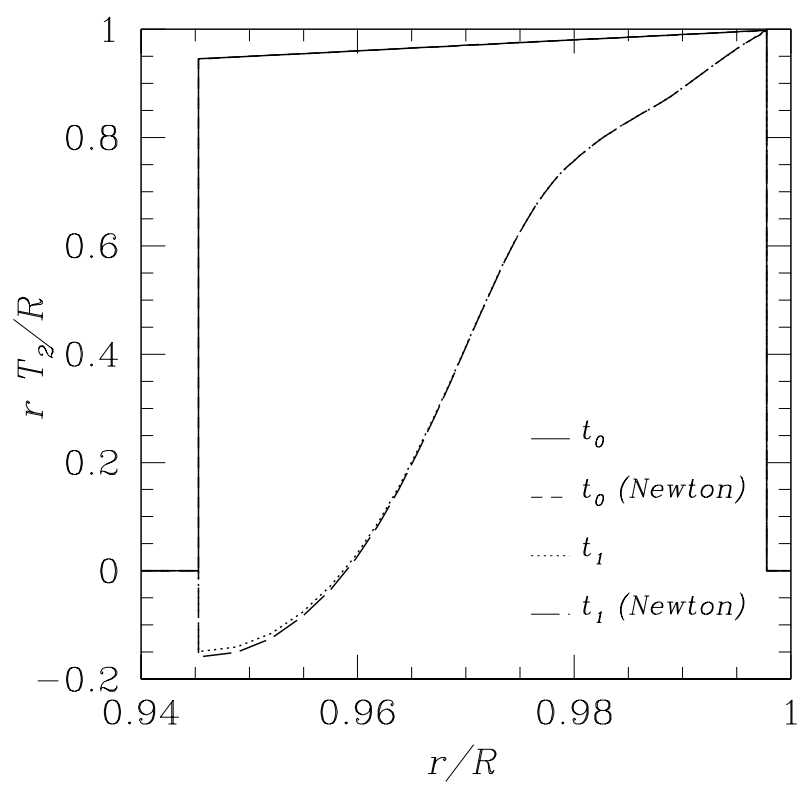

Fig. 8. Displacement vector $r T_{2} / R$ of the $t_{0}$ and the $t_{1}$ modes of the neutron star model NS13T8, given as a function of $r / R$. Here, normalization of the eigenfunction is chosen as $T_{2}\left(R_{\text {out }}\right)=1$, where $R_{\text {out }}$ is the radius at the crust/ocean interface. The Newtonian eigenfunctions are shown as well as the relativistic ones. Note that two curves for $r T_{2} / R$ nearly overlap each other.

not strongly affected by introducing general relativity in the sense that the relativistic eigenfunctions of a mode have almost the same properties as the corresponding Newtonian eigenfunctions. An exception may be the two interfacial modes whose eigenfunctions differ from their Newtonian eigenfunctions, and we have shown that this can be explained as a result of the mode exchange between the two modes through an avoided crossing that occurs when we move from Newtonian dynamics to relativistic dynamics. 


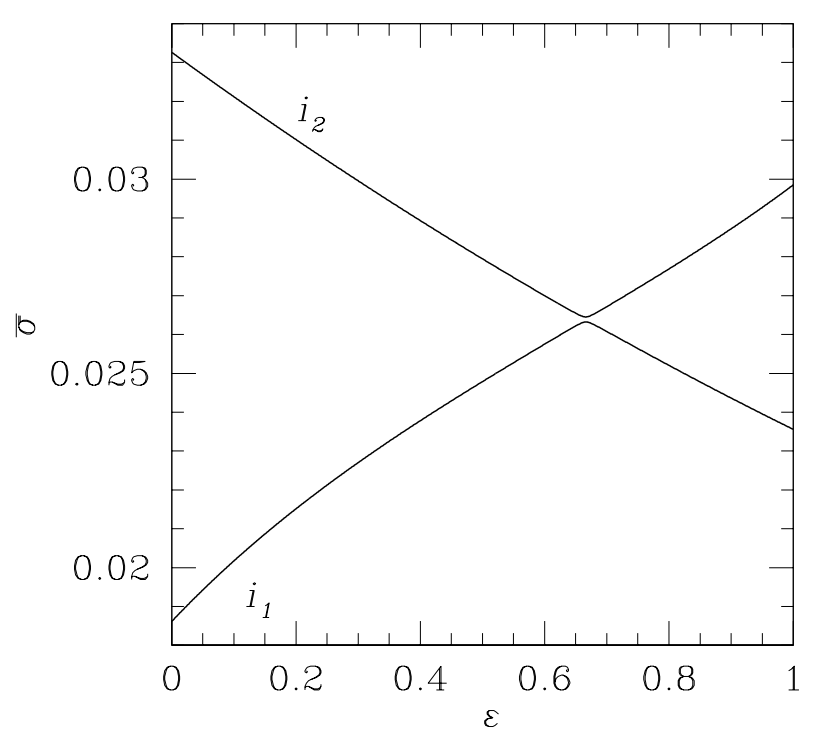

Fig. 9. The avoided crossing between the $i_{1}$ and $i_{2}$ mode of the model NS13T8, where $\varepsilon$ is the parameter that represents the strength of general relativistic effects. The avoided crossing happens at $\varepsilon \sim 0.67$.

Needless to say, nonradial oscillations of neutron stars should be treated within the framework of general relativity because of their strong gravity. At present, however, we cannot investigate the pulsations of neutron stars in all the aspects by using fully general relativistic formalism because of the complexity general relativity brings about ${ }^{2}$. One of the simplest ways to study the oscillations of a neutron star is to treat the problems within the framework of Newtonian dynamics, that is, to solve the Newtonian oscillation equations for neutron star models constructed with the Newtonian hydrostatic equations. However, it is obvious that this Newtonian treatment cannot be fully approved for neutron stars since the Newtonian equilibrium models do not give us correct mass and radius for the stars. To reduce the distance between fully relativistic and Newtonian calculations of nonradial oscillations of the stars, we may use relativistic equilibrium models and solve the Newtonian oscillation equations, which was the strategy taken by McDermott et al. (1988). In this paper, as an extension of the study by McDermott et al. (1988), we solved relativistic oscillation equations derived in the relativistic Cowling approximation for relativistic neutron star models with a solid crust. Our calculation confirmed that the classification scheme McDermott et al. (1988) employed for the oscillation modes of neutron stars with a solid crust is valid even if we integrate relativistic oscillation equations.
Acknowledgements. S.Y. acknowledges financial support from the Portuguese FCT through a Sapiens project, number 36280/99.

\section{References}

Aki, K., \& Richards, P. G. 1980, Quantitative Seismology: Theory and Methods (Freeman, San Francisco)

Andersson, N. 1998, ApJ, 502, 708

Andersson, N., Kojima, Y., \& Kokkotas, K. D. 1996, ApJ, 462, 855

Andersson, N., \& Kokkotas, K. D. 2001, Int. J. Mod. Phys. D, 10, 381

Andersson, N., Kokkotas, K. D., \& Schutz, B. F. 1995, MNRAS, 274, 1039

Carter, B., \& Quintana, H. 1972, Proc. R. Soc. Lond. A, 331, 57

Friedman, J. L. 1978, Commun. Math. Phys., 62, 247

Friedman, J. L., \& Morsink, S. M. 1998, ApJ, 502, 714

Friedman, J. L., \& Schutz, B. F. 1975, ApJ, 200, 204

Finn, L. S. 1990, MNRAS, 245, 82

Gudmundsson, E. H., Pethick, C. J., \& Epstein, R. I. 1983, ApJ, 272, 286

Kojima, Y. 1998, MNRAS, 293, 49

Kojima, Y., \& Hosonuma, M. 2000, Phys. Rev. D, 62, 044006

Lee, U., \& Strohmayer, T. E. 1996, A\&A, 331, 155

Leins, M., Nollert, H. P., \& Soffel, M. H. 1993, Phys. Rev. D, 48, 3467

Lindblom, L., \& Detweiler, S. L. 1983, ApJS, 53, 73

Lindblom, L., \& Splinter, R. J. 1990, ApJ, 348, 198

Lockitch, K. H., Andersson, N., \& Friedman, J. L. 2001, Phys. Rev. D, 63, 024019

McDermott, P. N., van Horn, H. M., \& Hansen, C. J. 1988, ApJ, 325, 725

McDermott, P. N., van Horn, H. M., \& Scholl, J. F. 1983, ApJ, 268, 837

Price, R. H., \& Thorne, K. S. 1969, ApJ, 155, 163

Regge, T., \& Wheeler, J. A. 1957, Phys. Rev., 108, 1063

Richardson, M. B., van Horn, H. M., Ratcliff, K. F., \& Malone, R. C. 1982, ApJ, 255, 624

Ruoff, J., \& Kokkotas, K. D. 2001, MNRAS, 328, 678

Schumaker, B. L., \& Thorne, K. S. 1983, MNRAS, 203, 457

Strohmayer, T. E. 1991, ApJ, 372, 573

Thorne, K. S. 1969a, ApJ, 158, 1

Thorne, K. S. 1969b, ApJ, 158, 997

Thorne, K. S. 1980, Rev. Mod. Phys., 52, 299

Thorne, K. S., \& Campolattaro, A. 1967, ApJ, 149, 591

Unno, W., Osaki, Y., Ando, H., Saio, H., \& Shibahashi, H. 1989, Nonradial Oscillations of Stars, second edition (University of Tokyo Press, Tokyo)

Yoshida, S. 2001, ApJ, 546, 1121

Yoshida, S., \& Kojima, Y. 1997, MNRAS, 289, 117

Yoshida, S., \& Lee, U. 2001, ApJ, 546, 1121

Yoshida, S., \& Lee, U. 2002, ApJ, 567, 1112

\footnotetext{
${ }^{2}$ One of the problems that appear in general relativistic formalism is the existence of gravitational waves and a necessity to deal with a complex eigenvalue problem corresponding to quasi-normal modes. Some authors have treated this problem (e.g., Lindblom \& Detweiler 1983; Leins et al. 1993; Andersson et al. 1995).
} 